\title{
OPEN Mortality prediction using a novel combination of biomarkers in the first day of sepsis in intensive care units
}

\author{
Junkun Liu ${ }^{2}$, Chengwen Bai ${ }^{1,4}$, Binbin $\mathrm{Li}^{1,4}$, Aijun Shan ${ }^{1 凶}$, Fei Shi ${ }^{1 凶}$, Can Yao ${ }^{1}$, Yu Zhang $^{1}$, \\ Jin Wang ${ }^{1}$, Weibu Chen ${ }^{3}$, Manying Xie ${ }^{1}$ \& Dehui Deng ${ }^{3}$
}

Early identification of infection severity and organ dysfunction is crucial in improving outcomes of patients with sepsis. We aimed to develop a new combination of blood-based biomarkers that can early predict 28-day mortality in patients with sepsis or septic shock. We enrolled 66 patients with sepsis or septic shock and compared 14 blood-based biomarkers in the first $24 \mathrm{~h}$ after ICU admission. The serum levels of interleukin-6 (IL-6) (median $217.6 \mathrm{vs.} 4809.0 \mathrm{pg} / \mathrm{ml}, P=0.001$ ), lactate (median 2.4 vs. $6.3 \mathrm{mmol} / \mathrm{L}, P=0.014), \mathrm{N}$-terminal prohormone of brain natriuretic peptide (NT-proBNP) (median 1596.5 vs. $32,905.3 \mathrm{ng} / \mathrm{ml}, P<0.001$ ), prothrombin time (PT) (median $15.6 \mathrm{vs} .20 .1 \mathrm{~s}$, $P=0.030$ ), activated partial thrombin time (APTT) (median $45.1 \mathrm{vs} .59 .0 \mathrm{~s}, P=0.026$ ), and international normalized ratio (INR) (median 1.3 vs. 1.8, $P<0.001$ ) were significantly lower in the survivor group. IL-6, NT-proBNP, and INR provided the best individual performance in predicting 28-day mortality of patients with sepsis or septic shock. Furthermore, the combination of these three biomarkers achieved better predictive performance (AUC $0.890, P<0.001$ ) than conventional scoring systems. In summary, the combination of IL-6, NT-proBNP, and INR may serve as a potential predictor of 28-day mortality in critically ill patients with sepsis or septic shock.

Sepsis is defined as life-threatening organ dysfunction caused by a dysregulated host response to infection ${ }^{1}$. It is a pathological syndrome caused by definite or suspected infection, and carries more than the mortality rates of $30 \%{ }^{2}$. So far, it remains one of the leading causes of death worldwide in people of all ages, and the pathophysiological processes of this disease are well accepted. It involves not only the complex effects of systemic inflammation and immune dysfunction, but also acute failure of multiple organ systems in the body ${ }^{3}$. The immune response to infective agents may trigger a cascade of cytokines like interleukin-6 (IL-6), causing cells impairment, organs failure, and ultimately result in the coagulopathy, which is significantly correlated to the prognosis of sepsis patients ${ }^{4}$. As the condition deteriorates, hypotension and tachycardia can occur due to cardiovascular disorders, and serum levels of N-terminal prohormone of brain natriuretic peptide (NT-proBNP) are seen to be elevated in patients with myocardial depression, and often associated with a poor prognosis ${ }^{5,6}$. Moreover, it has been shown that serum levels of lactate and procalcitonin (PCT), Sequential Organ Failure Assessment (SOFA), and Acute Physiology and Chronic Health Evaluation II (APACHE II) scores are predictive of fatal outcomes in patients with critical illness ${ }^{7}$. Currently, there is still a lot of ambiguity surrounding the risk stratification and prognostic evaluation of comprehensive indicators for sepsis. Therefore, it may provide great value for patients with sepsis by establishing an accurate multi-index joint detection system.

The severity of infection and reversibility of organ dysfunction strongly affect the outcomes of sepsis patients. Although some studies have evaluated the prognostic value of some biomarkers such as lactate ${ }^{8,9}$, most of them focused only on inflammation or one organ failure, and the comprehensive assessment of biomarkers involving inflammatory states and different organ functions was rarely performed. In this study, we demonstrate

\footnotetext{
${ }^{1}$ Emergency Department, Shenzhen People's Hospital (The First Affiliated Hospital of Southern University of Science and Technology, The Second Clinical Medical College of Jinan University), Shenzhen, Guangdong, China. ${ }^{2}$ Dermatology Department, Xiangya Medical College, Central South University, Changsha, Hunan, China. ${ }^{3}$ Laboratory Department, Shenzhen People's Hospital (The First Affiliated Hospital of Southern University of Science and Technology, The Second Clinical Medical College of Jinan University), Shenzhen, Guangdong, China. ${ }^{4}$ These authors contributed equally: Chengwen Bai and Binbin Li. ${ }^{\square}$ email: shanaij@yeah.net; shi.fei@ szhospital.com
} 


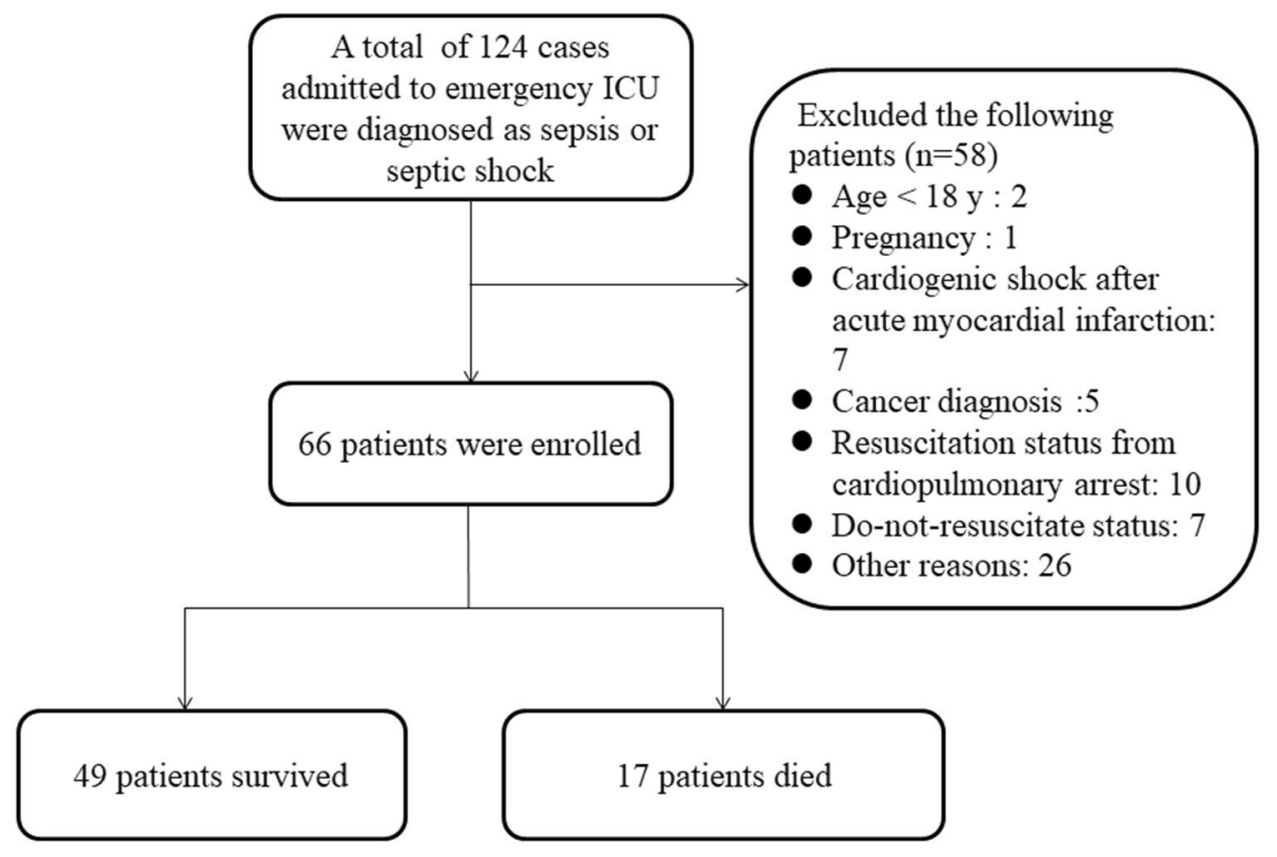

Figure 1. Screening and enrollment of the study.

the prognostic significance of several serological indicators such as IL-6, PCT, NT-proBNP, lactate, hepatorenal function, and coagulation function, to provide a clinical screening tool to identify and initiate early management of patients with sepsis.

\section{Materials and methods}

This prospective, observational study was conducted on patients directly admitted to the Emergency intensive care unit (ICU) via Emergency Room, Shenzhen People's Hospital, the first affiliated hospital of Southern University of Science and Technology, the second clinical medical college of Jinan University, from April 2017 to December 2018. In addition, all patients and/or family members signed informed consent and in accordance with the Ethical Guidelines of the Helsinki Declaration. This study was carried out with the approval of the Ethics Committee of Shenzhen People's Hospital, the Second Clinical Medical College of Jinan University (NO. LL-KT-201705001).

Study population. Adult patients ( $\geq 18$ years) admitted for sepsis or septic shock, according to Sepsis-3 criteria, were enrolled within $24 \mathrm{~h}$ of admission to the emergency ICU (Fig. 1). Following cases were excluded from the study: (1) under 18 years of age, (2) pregnancy, (3) shock of other origin, (4) acute coronary syndrome, (5) cancer, (6) resuscitation status from cardiopulmonary arrest, (7) trauma, (8) a requirement for immediate surgical intervention, (9) antibacterial therapy in the 5 days prior to enrollment, (10) a transfer to another hospital within $24 \mathrm{~h}$ after ICU admission, (11) having a slow progression of the disease over 7 days, and (12) those on do-not-resuscitate status. All subjects were given timely initial interventions, including appropriate antibiotics, hemodynamic resuscitation, and organ support therapy using protective mechanical ventilation or hemodialysis according to the Surviving Sepsis Campaign (SSC) 2016 guideline recommendations.

Biomarker measurements. Blood samples were collected during first $24 \mathrm{~h}$ at ICU admission, and stored frozen until biomarker assays were performed (per manufacturer's instructions) in the Laboratory Department of Shenzhen People's Hospital. The serum levels of 14 biomarkers were assayed: immunoturbidimetry method (Roche) for high-sensitivity C-reactive protein (hs-CRP), enzyme-linked fluorescent assay (VIDAS) for PCT, troponin I (Tn-I) and D-dimer, electro-chemiluminescence immunoassay (Roche) for IL-6 and NTproBNP, colorimetric assay (Roche) for serum lactate, enzymatic colorimetric method (Roche) for serum creatinine (Cr), colorimetric diazo method (Roche) for total bilirubin, impedance and single light-scatter method for platelet (PLT) counting, coagulation tests (Sysmex) for prothrombin time (PT), activated partial thrombin time (APTT) and fibrinogen (Fib), and international normalized ratio (INR) was calculated from the following formula: INR = patient PT/mean normal PT) $\wedge$ International Sensitivity Index (ISI). If the levels of biomarkers were measured multiple times in the first $24 \mathrm{~h}$, the average level of each biomarker was used in our study.

Data collection. We collected the following data: baseline demographic information, comorbidities, use of vasopressors, use of mechanical ventilator (MV), use of dialysis, length of hospital and ICU stay; mortality in 28 days; clinical indicators including mean arterial blood pressure (MAP), Glasgow Coma Scale (GCS) $)^{10}$, SOFA $^{11}$, and APACHEII scores ${ }^{12}$. These scoring systems were calculated based on the data obtained during 
the first $24 \mathrm{~h}$ after ICU admission. Furthermore, the baseline pulse-induced contour cardiac output (PICCO) parameters of cardiac preload including the intrathoracic blood volume (ITBV) and global end diastolic volume (GEDV) were also collected. The follow-up phone calls were conducted with all subjects discharged from the hospital to collect the information for assessing 28-day mortality.

Statistical evaluation. Statistical Package for Social Sciences (SPSS), version 19.0 (SPSS Inc, Chicago, IL) was used for statistical evaluations, and then the normal distribution of the data was confirmed by Kolmogorov-Smirnov test. Normally and non-normally distributed continuous variables were summarized as the mean (standard deviation, SD) and as the median (interquartile range, IQR), respectively. Data was analyzed by T test, post hoc LSD test or Mann-Whitney U test when applicable. The chi-squared test was for categorical variables. The univariate and multivariate logistic regression analyses were used to analyze the independent effects of various parameters on 28-day mortality. In order to identify the correlation between blood biomarkers and APACHEII or SOFA, variables were analysed using the Pearson correlation coefficients. The Receiver operating characteristic (ROC) curves were performed to predict the association of biomarkers with 28-day mortality in patients with sepsis ${ }^{13}$. A $P$ value of less than $0.05(P<0.05)$ was considered as a statistically significant difference.

\section{Results}

Baseline characteristics and outcomes. A total of 66 patients with sepsis or septic shock were enrolled in this study after screened for eligibility. According to 28-day follow-up, 66 patients with sepsis were divided into survivor group $(\mathrm{n}=49,30$ males and 19 females), and non-survivor group $(\mathrm{n}=17,9$ males and 8 females). Among them, the average length of hospital and ICU stay were 11.4 \pm 8.4 days and $5.5 \pm 5.8$ days respectively. The 28 -day overall mortality was $25.8 \%$. Inter-group comparisons revealed statistically significant differences in APACHE II (non-survivors vs. survivors: $36.5 \pm 7.6$ vs. $25.1 \pm 8.8, P<0.001$ ), SOFA (non-survivors vs. survivors: $9.9 \pm 4.6$ vs. $6.2 \pm 3.6, P=0.001$ ), and GCS scores (non-survivors vs. survivors: $7.1 \pm 4.2$ vs. $11.8 \pm 4.4, P<0.001$ ). Furthermore, non-survivors had a greater risk of cardiovascular disease as a previous comorbidity (non-survivors vs. survivors: $47.1 \%$ vs. $18.4 \%, P=0.020$ ), and had a higher probability of mechanical ventilation (nonsurvivors vs. survivors: $64.7 \%$ vs. $20.4 \%, P=0.001$ ). While age, gender, MAP, etiology, and duration of hospital stay showed no statistical differences between the two groups. The comparisons between survivors and nonsurvivors are depicted in Table 1.

Comparison of blood-based biomarkers between survivor and non-survivor groups. The serum levels of IL-6 [survivors vs. non-survivors: $217.6(103.3-962.4)$ vs. $4809.0(247.2-5000.0) \mathrm{pg} / \mathrm{ml}, P=0.001$ ], lactate [survivors vs. non-survivors: $2.4(1.8-3.2)$ vs. $6.3(2.5-14.3) \mathrm{mmol} / \mathrm{L}, P=0.014$ ], and NT-proBNP [survivors vs. non-survivors: $1596.5(1708.6-10,635.4)$ vs. $32,905.3(17,942.5-35,000.0) \mathrm{ng} / \mathrm{ml}, P<0.001]$ were significantly lower in survivor group when compared to the non-survivor group, as shown in Table 2. There were no differences in serum levels of hs-CRP, PCT, Tn-I, Cr, and total bilirubin $(P=0.849,0.961,0.953,0.215,0.505$, respectively) between the two groups. Since sepsis frequently is accompanied by coagulation abnormalities, we also compared coagulation function between non-survivors and survivors. We found that non-survivors had higher levels of PT [non-survivors vs. survivors: 20.1 (16.9-25.2) vs. $15.6(13.2-17.4) \mathrm{s}, P=0.030$ ], APTT [nonsurvivors vs. survivors: $59.0(46.8-90.5)$ vs. $45.1(38.5-55.3)$ s, $P=0.026$ ], and INR [non-survivors vs. survivors: $1.8(1.5-3.8)$ vs. $1.3(1.1-1.5), P<0.001]$ as compared to survivors. There were no significant differences with respect to other coagulation function indicators, including PLT, Fib, and D-dimer levels $(P=0.919,0.882,0.717$, respectively).

Logistic regression analysis of blood-based biomarkers associated with 28-day mortality. In this study, the variables of IL-6, lactate, NT-proBNP, PT, APTT, and INR for which $P<0.05$ between non-survivors and survivors were included. In the logistic regression analysis, the levels of IL-6 [odds ratio (OR) 1.001; 95\% confidence interval (CI) 1.000-1.001; $P=0.010$ ], NT-proBNP (OR 1.000; 95\% CI 1.000-1.000; $P=0.013$ ), and INR (OR 1.917 ; 95\% CI $0.949-3.871 ; P=0.046)$ within the first $24 \mathrm{~h}$ of admission were independently associated with 28-day mortality of sepsis (Table 3 ), and were valuable for predicting 28 -day mortality. However, other variables including lactate, PT, and APTT were not significant predictors of 28-day mortality.

ROC curve analysis of blood-based biomarkers for predicting 28-day mortality. The ability of selected biomarkers, SOFA, and APACHE II scores to predict the 28-day mortality in patients with sepsis according to ROC curve analysis was shown in Table 4 and Fig. 2. The optimal cutoff value for IL-6 was $2580.5 \mathrm{pg} /$ $\mathrm{ml}$, with sensitivity of $63 \%$, and specificity of $90 \%$ (AUC $0.785,95 \%$ CI $0.647-0.923, P=0.001$ ); $3.75 \mathrm{mmol} / \mathrm{l}$ for lactate with $63 \%$ sensitivity and $84 \%$ specificity (AUC $0.776,95 \%$ CI $0.633-0.918, P=0.001$ ); $9086.59 \mathrm{ng} / \mathrm{ml}$ for NT-proBNP with $70 \%$ sensitivity and $80 \%$ specificity (AUC $0.825,95 \%$ CI $0.704-0.947, P<0.001$ ); $17.55 \mathrm{~s}$ for PT with $71 \%$ sensitivity and $78 \%$ specificity (AUC $0.700,95 \%$ CI $0.529-0.870, P=0.017$ ); 44.50 s for APTT with $82 \%$ sensitivity and $47 \%$ specificity (AUC $0.698,95 \%$ CI $0.552-0.844, P=0.016$ ); and 1.465 for INR with $81 \%$ sensitivity and $76 \%$ specificity (AUC $0.748,95 \%$ CI $0.590-0.906, P=0.003$ ). Moreover, we also performed the combination tests to gain a better performance, and revealed that the combination of IL- 6 , NT-proBNP and INR (AUC $0.890,95 \%$ CI $0.791-0.989, P<0.001$ ) had superiority in predicting 28-day mortality than that of SOFA (AUC $0.732,95 \%$ CI $0.586-0.878, P=0.005$ ) and APACHE II scores (AUC 0.850, 95\% CI 0.783-0.942, $P<0.001$ ).

Correlation analysis of biomarkers in the study groups. The correlation coefficient analysis revealed a strong positive relationship between APACHEII and SOFA scores $(r=0.797, P<0.001)$. Furthermore, the levels 


\begin{tabular}{|c|c|c|c|c|}
\hline Characteristics & Survivor group $(n=49)$ & Non-survivor group $(n=17)$ & Total $(n=66)$ & $P$ value \\
\hline Age, median (IQR) & $69(54-82)$ & $77(63-84)$ & $73(55.8-82)$ & 0.099 \\
\hline Range & $26-97$ & $50-97$ & $26-97$ & \\
\hline Sex, male, n (\%) & $30(61.2)$ & $9(52.9)$ & $39(59.1)$ & 0.606 \\
\hline BMI, mean (SD) & $24.5(2.3)$ & $23.8(2.2)$ & $24.4(2.2)$ & 0.766 \\
\hline MAP (mmHg), mean (SD) & $82.5(15.2)$ & $86.9(16.3)$ & $83.6(15.5)$ & 0.311 \\
\hline \multicolumn{5}{|l|}{ Comorbidity } \\
\hline Diabetes mellitus, n (\%) & $20(40.8)$ & $5(29.4)$ & $25(37.9)$ & 0.404 \\
\hline Hypertension, n (\%) & $25(51.02)$ & $11(64.70)$ & $36(54.5)$ & 0.329 \\
\hline Cardiovascular disease, $\mathrm{n}(\%)$ & $9(18.4)$ & $8(47.1)$ & $17(25.8)$ & $0.020^{*}$ \\
\hline Chronic renal failure, $\mathrm{n}(\%)$ & $17(34.7)$ & $7(41.2)$ & $24(36.4)$ & 0.632 \\
\hline Chronic lung disease, $\mathrm{n}(\%)$ & $6(12.2)$ & $3(17.6)$ & $9(13.6)$ & 1.0 \\
\hline Use of vasopressors, $n$ (\%) & $6(12.2)$ & $3(17.6)$ & $9(13.6)$ & 0.881 \\
\hline Use of mechanical ventilation, $n(\%)$ & $10(20.4)$ & $11(64.7)$ & $21(31.8)$ & $0.001^{*}$ \\
\hline Use of dialysis, $\mathrm{n}(\%)$ & $4(8.2)$ & $4(23.5)$ & $8(12.1)$ & 0.214 \\
\hline \multicolumn{5}{|l|}{ Sepsis etiology } \\
\hline Pneumonia, n (\%) & $32(65.3)$ & $14(82.4)$ & $46(69.7)$ & 0.312 \\
\hline Urinary tract infection, $\mathrm{n}(\%)$ & $20(40.8)$ & $3(17.6)$ & $23(34.8)$ & 1.152 \\
\hline Intra-abdominal infection, $\mathrm{n}(\%)$ & $9(18.4)$ & $3(17.6)$ & $12(18.2)$ & 1.0 \\
\hline Soft tissue infection, $\mathrm{n}(\%)$ & $3(6.1)$ & $0(0)$ & $3(4.5)$ & 0.563 \\
\hline \multicolumn{5}{|l|}{ Clinical scoring } \\
\hline SOFA, mean (SD) & $6.2(3.6)$ & $9.9(4.6)$ & $7.2(4.2)$ & $0.001^{*}$ \\
\hline APACHE II score, mean (SD) & $25.1(8.8)$ & $36.5(7.6)$ & $28.1(9.9)$ & $0.000^{*}$ \\
\hline GCS, mean (SD) & $11.8(4.4)$ & $7.1(4.2)$ & $10.6(4.8)$ & $0.000^{*}$ \\
\hline \multicolumn{5}{|l|}{ PICCO parameters } \\
\hline $\operatorname{GEDI}\left(\mathrm{ml} / \mathrm{m}^{2}\right)$, mean $(\mathrm{SD})$ & $764.7(234.0)$ & $779.5(75.0)$ & $770.6(179.9)$ & 0.890 \\
\hline ITBV $\left(\mathrm{ml} / \mathrm{m}^{2}\right)$, mean $(\mathrm{SD})$ & $914.5(282.5)$ & $915.0(97.1)$ & $914.7(217.9)$ & 0.997 \\
\hline Hospital stay (day), mean (SD) & $12.1(7.1)$ & $9.5(11.6)$ & $11.4(8.4)$ & 0.392 \\
\hline
\end{tabular}

Table 1. Demographic characteristics at ICU admission. IQR range interquartile, $B M I$ body mass index, $M A P$ mean arterial pressure, SOFA sequential organ failure assessment, GCS Glasgow coma scale, APACHE II acute physiology and chronic health score II, PICCO Pulse-induced contour cardiac output, GEDI Global end-diastolic blood volume, ITBV Intrathoracic blood volume, ICU intensive care unit. *Indicates a significant value, $P<0.05$.

of IL-6, NT-proBNP, INR, lactate and PT in all patients was positively associated with APACHEII $(\mathrm{r}=0.492$, $0.535,0.471,0.371,0.471$, respectively; $P$ all $<0.05)$ and SOFA scores $(\mathrm{r}=0.495,0.572,0.288,0.347,0.355$, respectively; $P$ all $<0.05)$. The APTT levels were positively associated with APACHEII scores $(\mathrm{r}=0.298, P=0.015)$. However, we didn't find an association between APTT levels and SOFA scores $(P=0.222)$. More details were shown in Table 5 and Fig. 3.

\section{Discussion}

We evaluated the inflammatory and organ-specific biomarkers to predict 28-day mortality for critically ill patients with sepsis, and found that a novel combination of INR, NT-proBNP and IL- 6 within first $24 \mathrm{~h}$ of ICU admission may rapidly predict mortality risk in patients with sepsis or septic shock. This combination of three markers is solely based on cardiac dysfunction, coagulation disorders, and inflammatory responses, which are readily available during the patient's course of ICU stay. Therefore, it may help improve risk stratification, early assessment and intervention of sepsis patients in ICU. Our findings also suggest that the optimal combination of INR, NT-proBNP and IL-6 has a significantly higher AUC value than that of conventional scoring systems such as SOFA and APACHEII scores, and provides need to confirm its validity for screening sepsis-related mortality.

Sepsis is also associated with coagulopathy ranging from hypercoagulability to acute disseminated intravascular coagulation (DIC), which may be likely play a key role in multiple organ dysfunction syndrome (MODS) ${ }^{14}$. In particular, hemostasis-related parameter has been shown to be a predictor of poor outcomes in ICU patients. Thrombocytopenia occurs in up to $40 \%$ of patients on admission, and an INR $\geq 1.5$ is present in approximately two-thirds of patients. We found that the INR value in survivors was significantly lower than in non-survivors, and strongly associated with greater risk of serious illness. Furthermore, multivariate analysis demonstrated that INR level was an independent factor for critically ill patients with sepsis, and high INR (> 1.47) was significantly associated with increased mortality risk. In fact, because coagulopathy (INR $>1.5$ ) is defined as one of the critical characteristics in patients with sepsis according to sepsis 3.0 criteria, this definition would partly support our results ${ }^{15}$. However, some authors still questioned the use of INR as a management tool because INR value can be falsely increased, and not necessarily associated with risk of bleeding events ${ }^{16}$. Our study also showed that the 


\begin{tabular}{|c|c|c|c|c|}
\hline Blood biomarkers & Survivor group $(n=49)$ & Nor-survivor group $(n=17)$ & Total $(n=66)$ & $P$ value \\
\hline \multicolumn{5}{|l|}{ Inflammation } \\
\hline hs-CRP (mg/L), median (IQR) & $188.3(102.9-295.7)$ & $175.7(136.5-300.2)$ & $187.9(129.8-296.8)$ & 0.849 \\
\hline PCT (ng/ml), median (IQR) & $28.0(6.1-95.3)$ & $29.0(10.8-102.4)$ & $28.5(6.3-97.1)$ & 0.961 \\
\hline IL-6 (pg/ml), median (IQR) & $217.6(103.3-962.4)$ & $4809.0(247.2-5000.0)$ & $313.7(121.3-2565.3)$ & $0.001^{*}$ \\
\hline \multicolumn{5}{|l|}{ Circulation } \\
\hline Lactate (mmol/l), median (IQR) & $2.4(1.8-3.2)$ & $6.3(2.5-14.3)$ & $2.5(1.9-4.1)$ & $0.014^{*}$ \\
\hline NT-proBNP (ng/ml), median (IQR) & $1596.5(1708.6-10,635.4)$ & $32,905.3(17,942.5-35,000.0)$ & $3720.1(880.9-23,665.7)$ & $<0.001^{*}$ \\
\hline Tn-I (ng/ml), median (IQR) & $0.0(0.01-0.12)$ & $0.2(0.01-0.98)$ & $0.01(0.01-0.31)$ & 0.953 \\
\hline \multicolumn{5}{|l|}{ Renal function } \\
\hline $\operatorname{Cr}(\mu \mathrm{mol} / \mathrm{l})$, median $(\mathrm{IQR})$ & $125.0(92.0-247.0)$ & $252.0(239.0-463.0)$ & $170.0(103.0-281.0)$ & 0.215 \\
\hline \multicolumn{5}{|l|}{ Liver function } \\
\hline Total bilirubin $(\mu \mathrm{mol} / \mathrm{l})$, median (IQR) & $13.8(5.0-70.9)$ & $14.8(8.3-110.9)$ & $14.0(6.0-88.9)$ & 0.505 \\
\hline \multicolumn{5}{|l|}{ Coagulation function } \\
\hline PLT $\left(\times 10^{9} / 1\right)$, mean $(\mathrm{SD})$ & $170.6(109.5)$ & $167.4(110.5)$ & $169.7(108.0)$ & 0.919 \\
\hline PT (s), median (IQR) & $15.6(13.2-17.4)$ & $20.1(16.9-25.2)$ & $16.2(14.0-19.5)$ & $0.030^{*}$ \\
\hline APTT (s), median (IQR) & $45.1(38.5-55.3)$ & $59.0(46.8-90.5)$ & $49.0(42.2-58.0)$ & $0.026^{*}$ \\
\hline Fib $(\mathrm{g} / \mathrm{l})$, median $(\mathrm{IQR})$ & $5.1(4.2-7.7)$ & $5.7(2.2-6.2)$ & $5.1(4.1-7.3)$ & 0.882 \\
\hline INR, median (IQR) & $1.3(1.1-1.5)$ & $1.8(1.5-3.8)$ & $1.4(1.2-1.8)$ & $<0.001^{\star}$ \\
\hline D-Dimer $(\mu \mathrm{g} / \mathrm{l})$, median (IQR) & $3948.5(2093.5-7848.0)$ & $8889.4(4278.9-10,000.0)$ & $4765.9(2792.7-8716.9)$ & 0.717 \\
\hline
\end{tabular}

Table 2. Biomarkers in blood of the study groups. $I Q R$ range interquartile, $h s$ - $C R P$ high-sensitivity C-reactive protein, $P C T$ procalcitonin, $I L-6$ Interleukin 6, NT-proBNP N-terminal prohormone of brain natriuretic peptide, Tn-I Troponin I, Cr creatinine, PLT Platelet, $P T$ prothrombin time, APTT activated partial thrombin time, Fib fibrinogen, INR international normalized ratio. ${ }^{\star}$ Indicates a significant value, $P<0.05$.

\begin{tabular}{|l|l|l|l|l|l|l|}
\hline \multirow{2}{*}{ Variables } & \multicolumn{4}{l}{ Univariable analysis } & \multicolumn{2}{l|}{ Multivariable analysis } \\
\cline { 2 - 8 } & OR & $\mathbf{9 5 \%}$ CI & P value & OR & $\mathbf{9 5 \%}$ CI & P value \\
\hline INR & 2.825 & $1.326-6.020$ & $0.007^{*}$ & 1.917 & $0.949-3.871$ & $0.046^{*}$ \\
\hline NT-proBNP & 1.000 & $1.000-1.000$ & $0.000^{*}$ & 1.000 & $1.000-1.000$ & $0.013^{*}$ \\
\hline IL-6 & 1.001 & $1.000-1.001$ & $0.000^{*}$ & 1.001 & $1.000-1.001$ & $0.010^{*}$ \\
\hline Lactate & 1.327 & $1.103-1.597$ & $0.003^{*}$ & - & - & - \\
\hline PT & 1.135 & $1.030-1.251$ & 0.011 & - & - & - \\
\hline APTT & 1.037 & $1.006-1.070$ & 0.019 & - & - & - \\
\hline CRP & 1.000 & $0.995-1.005$ & 0.981 & - & - & - \\
\hline PCT & 0.999 & $0.990-1.009$ & 0.906 & - & - & - \\
\hline Tn-I & 0.995 & $0.833-1.187$ & 0.952 & - & - & - \\
\hline Cr & 1.002 & $0.999-1.004$ & 0.178 & - & - & - \\
\hline PLT & 0.896 & $0.995-1.009$ & 0.978 & & & \\
\hline Fib & 0.898 & $0.712-1.133$ & 0.363 & - & - & - \\
\hline D-Dimer & 1.000 & $1.000-1.000$ & 0.054 & - & - & - \\
\hline
\end{tabular}

Table 3. The logistic regression analysis predicting 28 -day mortality in patients with sepsis or septic shock. OR odds ratio, CI confidence interval, INR international normalized ratio, NT-proBNP N-terminal prohormone of brain natriuretic peptide, $I L-6$ Interleukin 6, PT prothrombin time, APTT activated partial thrombin time, $h s$-CRP high-sensitivity C-reactive protein, $P C T$ procalcitonin, $T n-I$ Troponin I, Cr creatinine, PLT Platelet, Fib fibrinogen. ${ }^{\star}$ Indicates a significant value, $P<0.05$.

INR value of 1.47 has a sensitivity of $81 \%$, specificity of $76 \%$ and AUC of 0.748 , which may limit its use alone at accurate assessment on the outcomes of sepsis patients.

Since NT-proBNP is mainly secreted by cardiomyocytes in response to increased ventricular volume or pressure load, the level of this biomarker is known to be highly sensitive to detect acute heart failure, and correlated with the severity of heart failure ${ }^{17,18}$. In addition, low-grade systematic inflammation and insulin resistance are other possible causes of elevated level of NT-proBNP in patients with sepsis ${ }^{19}$. Previous studies revealed the levels of NT-proBNP in sepsis patients without heart failure were associated with in-hospital mortality ${ }^{20}$. Therefore, it has been accepted that the increased levels of NT-proBNP in septic shock patients are mainly due to sepsis rather than cardiac insufficiency. In agreement with previous studies, we found that on the first day of ICU admission, the levels of NT-proBNP in non-survivor group were significantly higher than in survivor group, and the higher 


\begin{tabular}{|l|l|l|l|l|l|l|}
\hline Blood biomarkers & AUC & 95\% CI & Cut-off & Sensitivity & Specificity & $P$-value \\
\hline INR & 0.748 & $0.590-0.906$ & 1.465 & 0.81 & 0.76 & $0.003^{*}$ \\
\hline NT-proBNP (ng/ml) & 0.825 & $0.704-0.947$ & 9086.59 & 0.70 & 0.80 & $0.000^{*}$ \\
\hline IL-6 (pg/ml) & 0.785 & $0.647-0.923$ & 2580.5 & 0.63 & 0.90 & $0.001^{*}$ \\
\hline Lactate (mmol/l) & 0.776 & $0.633-0.918$ & 3.75 & 0.63 & 0.84 & $0.001^{*}$ \\
\hline PT (s) & 0.700 & $0.529-0.870$ & 17.55 & 0.71 & 0.78 & $0.017^{*}$ \\
\hline APTT (s) & 0.698 & $0.552-0.844$ & 44.50 & 0.82 & 0.47 & $0.016^{*}$ \\
\hline SOFA scores & 0.732 & $0.586-0.878$ & 7.5 & 0.71 & 0.76 & $0.005^{*}$ \\
\hline APACHE II scores & 0.850 & $0.758-0.942$ & 27.5 & 0.94 & 0.69 & $0.000^{*}$ \\
\hline IL-6+ NT-proBNP & 0.879 & $0.783-0.974$ & - & 0.75 & 0.92 & $0.000^{*}$ \\
\hline IL-6+ INR+ NT-proBNP & 0.890 & $0.791-0.989$ & - & 0.81 & 0.90 & $0.000^{*}$ \\
\hline
\end{tabular}

Table 4. Prognostic value of clinical parameters. AUC Area under the curve, $C I$ confidence interval, $I L-6$ Interleukin 6, INR international normalized ratio, NT-proBNP N-terminal prohormone of brain natriuretic peptide, $P T$ prothrombin time, $A P T T$ activated partial thrombin time, SOFA sequential organ failure assessment, APACHE II acute physiology and chronic health score II. ${ }^{\star}$ Indicates a significant value, $P<0.05$.

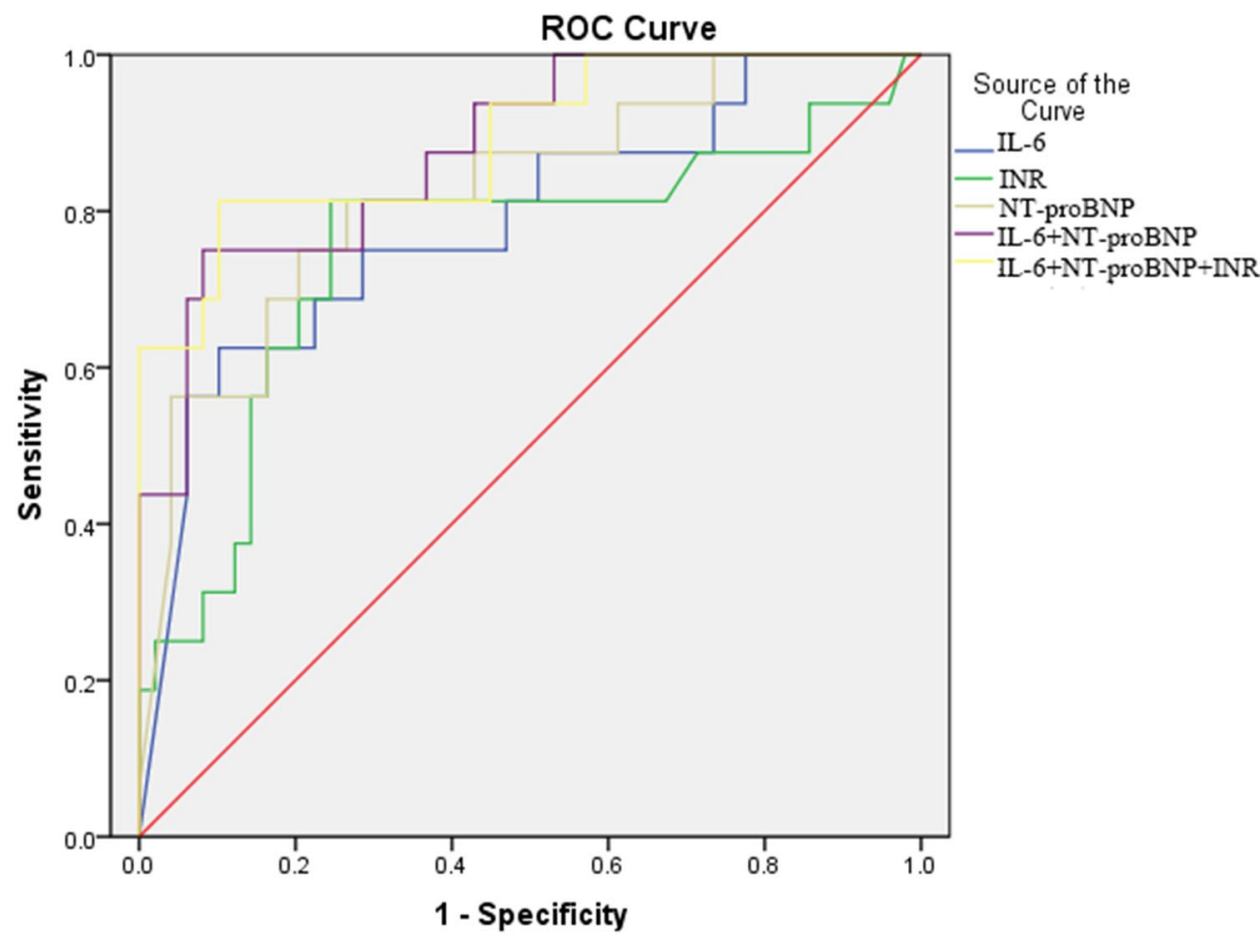

Figure 2. The receiver operating characteristic (ROC) curves of the selected biomarkers for predicting 28-day mortality of critically ill patients with sepsis or septic shock. The ROC curves for single biomarker and their combinations had the following areas: interleukin-6 (IL-6), 0.785; international normalized ratio (INR), 0.748; $\mathrm{N}$-terminal pro-brain natriuretic peptide (NT-proBNP), 0.825; combination of IL-6 and NT-proBNP, 0.879; and combination of IL-6, INR and NT-proBNP, 0.890.

NT-proBNP levels were associated with poorer health status, including higher SOFA scores and higher APACHE II scores. Moreover, the volume challenge during resuscitation process might be a possible reason for functional heart failure, and had some influence on the increased levels of NT-proBNP. In this study, we also found that NT-proBNP also presented as an independent risk factor for sepsis mortality. At a cut-off value of $9086.59 \mathrm{ng} /$ $\mathrm{ml}$, NT-proBNP had a sensitivity of $70 \%$ and a specificity of $80 \%$ with an AUC of 0.825 . These findings suggested NT-proBNP may be a potential predictor of sepsis mortality, but needs to increase its sensitivity and specificity by combining with other parameters.

Elevated levels of serum IL-6 have been found in both neonatal and adult sepsis ${ }^{21,22}$. Previous studies have shown that IL- 6 may be induced by infections and tissue injuries, and associated with a high risk of death in septic shock ${ }^{23}$. However, IL-6 is a multifunctional cytokine that has pro- and anti-inflammatory properties, and can be affected by different conditions such as exercise, aging, certain cancers, frailty, and chronic inflammatory response $^{24}$. These factors should be comprehensively considered for a better understanding of sepsis when IL-6 


\begin{tabular}{|l|l|l|l|l|}
\hline \multirow{2}{*}{ Blood biomarkers } & APACHE II & \multicolumn{2}{l|}{ SOFA } \\
\cline { 2 - 5 } & r value & P value & r value & P value \\
\hline IL-6 & 0.492 & $0.000^{*}$ & 0.495 & $0.000^{*}$ \\
\hline NT-proBNP & 0.535 & $0.000^{*}$ & 0.572 & $0.000^{*}$ \\
\hline INR & 0.471 & $0.000^{*}$ & 0.288 & $0.019^{*}$ \\
\hline Lactate & 0.371 & $0.002^{*}$ & 0.347 & $0.004^{*}$ \\
\hline PT & 0.471 & $0.000^{*}$ & 0.355 & $0.003^{*}$ \\
\hline APTT & 0.298 & $0.015^{*}$ & 0.152 & 0.222 \\
\hline
\end{tabular}

Table 5. Correlations between blood biomarkers and APACHE II or SOFA scores in all subjects. ${ }^{\star}$ Indicates a significant value, $P<0.05$. IL-6 Interleukin 6, INR international normalized ratio, NT-proBNP N-terminal prohormone of brain natriuretic peptide, $P T$ prothrombin time, APTT activated partial thrombin time, APACHE II acute physiology and chronic health score II, SOFA sequential organ failure assessment.
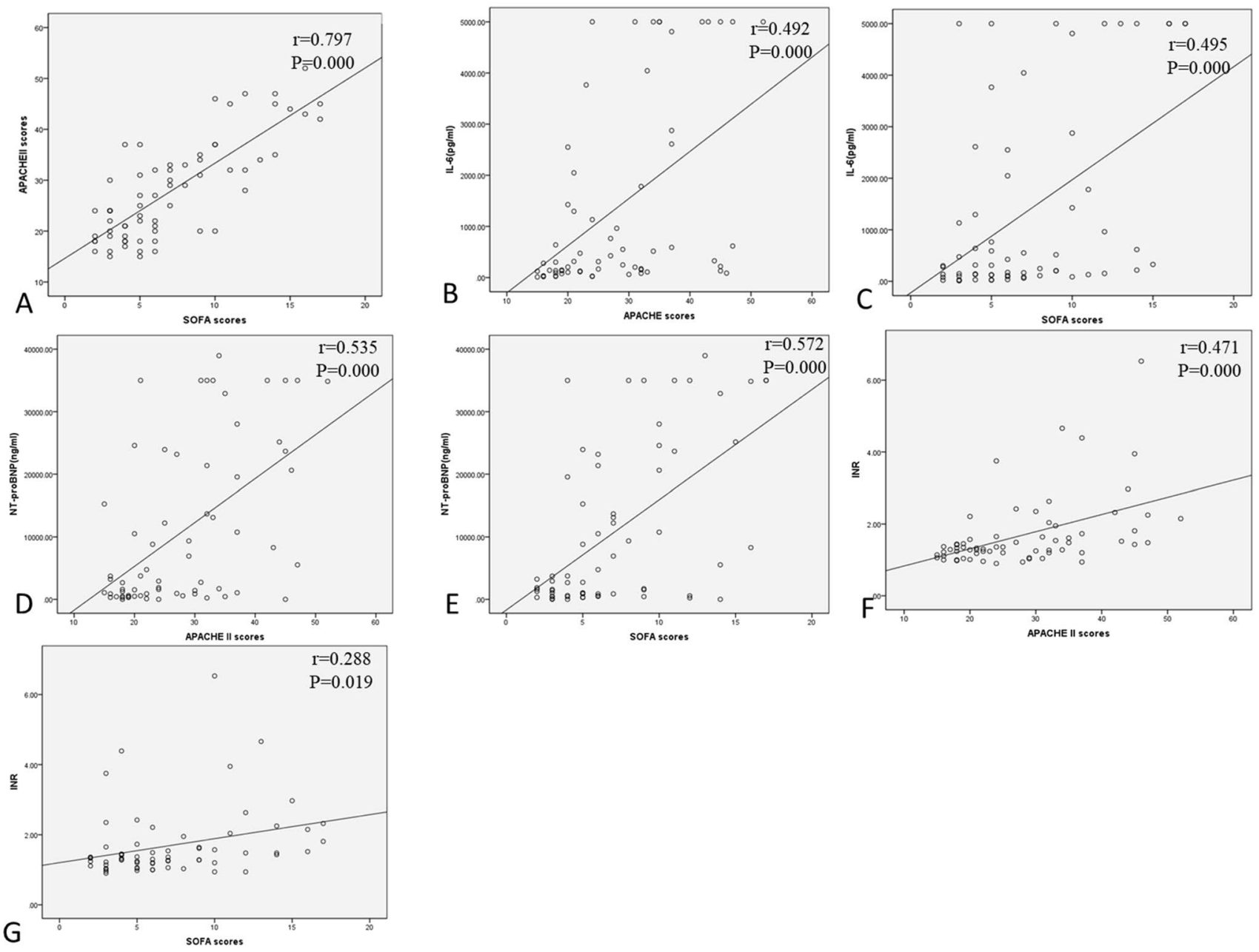

Figure 3. Correlation analysis of biomarkers in all subjects. (A) Correlation between APACHE II and SOFA, (B) Correlation between IL-6 and APACHE II, (C) Correlation between IL-6 and SOFA, (D) Correlation between NT-proBNP and APACHE II, (E) Correlation between NT-proBNP and SOFA, (F) Correlation between INR and APACHE II, and (G) Correlation between INR and SOFA.

levels are elevated. In this study, we observed that the IL-6 levels measured on day 1 were significantly increased in non-survivors when compared with survivors, and positively correlated with SOFA and APACHEII scores. A cut-off value for IL-6 on $2580.5 \mathrm{pg} / \mathrm{ml}$ was found to have a moderate sensitivity of $63 \%$ and a high specificity of $90 \%$ (AUC 0.785 ) for predicting mortality. The results suggested IL-6 may be used as a prognostic biomarker for 28-day mortality in patients with sepsis, while the predictive accuracy of IL-6 is lower than that of NT-proBNP.

Various studies have demonstrated that the APACHE II and SOFA scoring systems are good tools for predicting mortality in ICU patients ${ }^{25,26}$. Unlike APACHE II system, the SOFA was not initially designed to predict 
hospital mortality, while it has been broadly used in a range of applications such as organ dysfunction assessment, predicting hospital survival, and identifying non-ICU patients at risk of sepsis. Furthermore, the specificities of APACHE II and SOFA scores were found to be somewhat low in identifying the outcomes in different subgroups of ICU patients ${ }^{27}$. In the present study, we also revealed that in predicting 28 -day mortality, APACHE II score of $>27.5$, and SOFA score $>7.5$ performed at sensitivities of $94 \%$, and $71 \%$, and specificities of $69 \%$ and $76 \%$, respectively. Even though APACHE II score performed well in mortality risk stratification in the ICU patients with sepsis, it tended to a higher sensitivity and lower specificity, leading to an overestimation of mortality risk (and consequently, a higher burden on hospital costs). Moreover, the combination of three biomarkers, INR, NT-proBNP and IL-6 yielded an ROC value of 0.890 , which provided higher diagnostic accuracy (sensitivity of $81 \%$ and specificity of $90 \%$ ) than any single biomarker, APACHE II and SOFA scores. This result showed that the three-marker combination may have the best performance in predicting sepsis-related mortality. Therefore, instead of just screening APACHE II and SOFA scores during the initial $24 \mathrm{~h}$ in the ICU, we prefer to monitor the three-marker combination to predict the short outcome of sepsis patients. Once the patient meets the cut-off levels of the combination, we should closely observe the changes of multiple physiological systems, and frequently adjust medical therapies accordingly to reduce the risk of death. Furthermore, these three markers together may help to early stage the disease, predict prognosis and response of intervention in critically ill patients with sepsis.

Our study has several limitations. First, it was a single-center study, and the small size of study population is the major limitation. Therefore, larger observations of multiple centers should be carried out to verify the results. Second, we did not include other clinical parameters such as arterial blood gas analysis and white blood cell count, because these variables have not shown a sufficient predictive power for the outcomes of sepsis patients. Future studies need to elucidate the potential application of other clinical biomarkers for prognosis in sepsis, including but not limited to the parameters associated with dysfunction of major organs.

Despite the above limitations, our findings indicate the combination of INR, NT-proBNP and IL-6 may represent a valuable tool for predicting mortality risk in critically ill patients with sepsis or septic shock. Furthermore, this simple combination shows a higher prognostic accuracy for 28-day mortality than the SOFA or APACHE II scores, and could have a potential application in guiding early management of sepsis patients in ICU. Future studies are necessary to explore whether the combination can improve patient managements and outcomes in ICU.

\section{Data availability}

Some or all data, or code generated or used during the study are available from the corresponding author by request.

Received: 22 June 2020; Accepted: 14 December 2020

Published online: 14 January 2021

\section{References}

1. Rhodes, A. et al. Surviving sepsis campaign: International guidelines for management of sepsis and septic shock: 2016. Crit. Care Med. 45, 486-552 (2017).

2. Luhr, R., Cao, Y., Söderquist, B. \& Cajander, S. Trends in sepsis mortality over time in randomised sepsis trials: A systematic literature review and meta-analysis of mortality in the control arm, 2002-2016. Crit. Care. 23, 241 (2019).

3. Liu, Y., Han, N., Li, Q. \& Li, Z. Regulatory mechanisms underlying sepsis progression in patients with tumor necrosis factor-alpha genetic variations. Exp. Ther. Med. 12, 323-328 (2016).

4. Costa, R. T. et al. T helper type cytokines in sepsis: Time-shared variance and correlation with organ dysfunction and hospital mortality. Braz. J. Infect. Dis. 23, 79-85 (2019).

5. Guarracino, F., Bertini, P. \& Pinsky, M. R. Cardiovascular determinants of resuscitation from sepsis and septic shock. Crit. Care. 23, $118(2019)$.

6. Shojaee, M. et al. Pro-BNP versus MEDS score in determining the prognosis of sepsis patients; a diagnostic accuracy study. Emerg (Tehran). 6, e4 (2018).

7. Liu, Z. et al. Prognostic accuracy of the serum lactate level, the SOFA score and the qSOFA score for mortality among adults with sepsis. Scand. J. Trauma Resusc. Emerg. Med. 27, 51 (2019).

8. Innocenti, F. et al. Prognostic value of serial lactate levels in septic patients with and without shock. Intern. Emerg. Med. 14, 1321-1330 (2019).

9. Yu, G. et al. Utility of the early lactate area score as a prognostic marker for septic shock patients in the emergency department. Acute Crit. Care. 34, 126-132 (2019).

10. Teasdale, G. \& Jennett, B. Assessment of coma and impaired consciousness. A practical scale. Lancet 2, 81-84 (1974).

11. Raith, E. P. et al. Prognostic accuracy of the SOFA score, SIRS criteria, and qSOFA score for in-hospital mortality among adults with suspected infection admitted to the intensive care unit. JAMA 317, 290-300 (2017).

12. Knaus, W. A., Draper, E. A., Wagner, D. P. \& Zimmerman, J. E. APACHE II: A severity of disease classification system. Crit. Care Med. 13, 818-829 (1985).

13. Habibzadeh, F., Habibzadeh, P. \& Yadollahie, M. On determining the most appropriate test cut-off value: The case of tests with continuous results. Biochem. Med. (Zagreb). 26, 297-307 (2016).

14. Lelubre, C. \& Vincent, J. L. Mechanisms and treatment of organ failure in sepsis. Nat. Rev. Nephrol. 14, 417-427 (2018).

15. Singer, M. et al. The third international consensus definitions for sepsis and septic shock (Sepsis-3). JAMA 315, 801-810 (2016).

16. Attermann, J. Inaccuracy of international normalized ratio (INR) measurements and suggestions for improved WHO guidelines for calibration of reference preparations-A statistical study. J. ThrombHaemost. 1, 537-544 (2003).

17. Santaguida, P. L. et al. BNP and NT-proBNP as prognostic markers in persons with acute decompensated heart failure: A systematic review. Heart Fail. Rev. 19, 453-470 (2014).

18. McKie, P. M. \& Burnett, J. C. NT-proBNP: The gold standard biomarker in heart failure. J. Am. Coll. Cardiol. 68, 2437-2439 (2016).

19. Tettamanti, C., Hervet, T., Grabherr, S. \& Palmiere, C. Elevation of NT-proBNP and cardiac troponins in sepsis-related deaths: A forensic perspective. Int. J. Legal Med. 130, 1035-1043 (2016).

20. Shor, R. et al. BNP in septic patients without systolic myocardial dysfunction. Eur. J. Intern. Med. 17, 536-540 (2006).

21. Ganesan, P., Shanmugam, P., Sattar, S. B. \& Shankar, S. L. Evaluation of IL-6, CRP and hs-CRP as early markers of neonatal sepsis. J. Clin. Diagn. Res. 10, DC13-17 (2016). 
22. Hou, T., Huang, D., Zeng, R., Ye, Z. \& Zhang, Y. Accuracy of serum interleukin (IL)-6 in sepsis diagnosis: A systematic review and meta-analysis. Int. J. Clin. Exp. Med. 8, 15238-15245 (2015).

23. Jekarl, D. W. et al. Procalcitonin as a diagnostic marker and IL-6 as a prognostic marker for sepsis. Diagn. Microbiol. Infect. Dis. 75, 342-347 (2013).

24. Del Giudice, M. \& Gangestad, S. W. Rethinking IL-6 and CRP: Why they are more than inflammatory biomarkers, and why it matters. Brain Behav. Immun. 70, 61-75 (2018)

25. Godinjak, A. et al. Predictive value of SAPS II and APACHE II scoring systems for patient outcome in a medical intensive care unit. Acta Med. Acad. 45, 97-103 (2016).

26. Innocenti, F. et al. SOFA score in septic patients: Incremental prognostic value over age, comorbidities, and parameters of sepsis severity. Intern. Emerg. Med. 13, 405-412 (2018).

27. Wang, Y., Wang, D., Fu, J. \& Liu, Y. Predictive value of SOFA, qSOFA score and traditional evaluation index on sepsis prognosis. Zhonghua Wei Zhong Bing JiJiu Yi Xue. 29, 700-704 (2017).

\section{Author contributions}

Conceptualization: J.L., B.L. and F.S.; methodology: J.L., B.L., F.S. and C.B.; formal analysis: J.L.; writing-original draft: J.L. and A.S.; writing—review \& editing: B.L., F.S., C.B. and A.S.; supervision: C.B. and A.S.; investigation: C.Y., Y.Z., J.W., W.C., M.X. and D.D.; visualization: F.S. and C.Y.; data curation: C.Y., Y.Z., J.W., W.C., M.X. and D.D.; project administration: Y.Z. and A.S.

\section{Funding}

This study was funded by the Science and Technology Planning Project of Guangdong Province, China (Project Number: 2016A020215025), the Natural Science Foundation of Guangdong Province, China (Project Number: 2020A151501040), and Scientific Research and Cultivation Project of Shenzhen People's Hospital (Project Number: SYKYPY201912).

\section{Competing interests}

The authors declare no competing interests.

\section{Additional information}

Correspondence and requests for materials should be addressed to A.S. or F.S.

Reprints and permissions information is available at www.nature.com/reprints.

Publisher's note Springer Nature remains neutral with regard to jurisdictional claims in published maps and institutional affiliations.

(c) (i) Open Access This article is licensed under a Creative Commons Attribution 4.0 International License, which permits use, sharing, adaptation, distribution and reproduction in any medium or format, as long as you give appropriate credit to the original author(s) and the source, provide a link to the Creative Commons licence, and indicate if changes were made. The images or other third party material in this article are included in the article's Creative Commons licence, unless indicated otherwise in a credit line to the material. If material is not included in the article's Creative Commons licence and your intended use is not permitted by statutory regulation or exceeds the permitted use, you will need to obtain permission directly from the copyright holder. To view a copy of this licence, visit http://creativecommons.org/licenses/by/4.0/.

(c) The Author(s) 2021 